\title{
ON CORRELATION BETWEEN DIFFUSION RATE OF RADIATION DEFECTS AND DEPTH OF THEIR PENETRATION DURING ION IMPLANTATION
}

\section{E. L. PANKRATOV}

Nizhny Novgorod State University

23 Gagarin Avenue

Nizhny Novgorod, 603950

Russia

Nizhny Novgorod State Technical University

24 Minin Street

Nizhny Novgorod, 603950

Russia

e-mail: elp2004@mail.ru

\begin{abstract}
In this paper, we introduce a model for the redistribution and interaction of point radiation defects between themselves, as well as their simplest complexes in a material, taking into account the experimentally non-monotonicity of the distribution of the concentration of radiation defects. To take into account this non-monotonicity, the previously used model in the literature for the analysis of spatio-temporal distributions of the concentration of radiation defects was supplemented by the concentration dependence of their diffusion coefficient.
\end{abstract}

Keywords and phrases: ion implantation, model of radiation defects, analytical approach for modelling.

Received June 12, 2019

(C) 2019 Scientific Advances Publishers 


\section{Introduction}

In the present time influence of different types of radiation processing on semiconductors is intensively analyzing [1-3]. Based on the analysis several recommendations to increase of radiation resistance have been formulated [4-6].

In this paper, we analyze redistribution and interaction between point radiation defects, as well as their simplest complexes in materials after ion implantation. A modification of the previously proposed model [7] describing the redistribution and interaction of radiation defects between themselves is proposed with the aim of taking into account the experimentally revealed non-monotonicity of the distribution of the concentrations of these defects [8].

\section{Method of Solution}

We determine spatio-temporal distributions of concentrations of point defects by solution of the following system of equations [7, 9]:

$$
\left\{\begin{array}{l}
\frac{\partial I(x, t)}{\partial t}=\frac{\partial}{\partial x}\left[D_{I}(I, V, T) \frac{\partial I(x, t)}{\partial x}\right]-K_{I}(T) I^{2}(x, t)-K_{r}(T) I(x, t) V(x, t), \\
\frac{\partial V(x, t)}{\partial t}=\frac{\partial}{\partial x}\left[D_{V}(I, V, T) \frac{\partial V(x, t)}{\partial x}\right]-K_{V}(T) V^{2}(x, t)-K_{r}(T) I(x, t) V(x, t) .
\end{array}\right.
$$

Here $I(x, t)$ and $V(x, t)$ are the spatio-temporal distributions of concentrations of inter stitials and vacancies. The first term on the righthand side of Equations (1) describes the diffusion of point defects with the temperature-dependent temperature $T$ and the concentration of point defects by the diffusion coefficient $D_{\rho}(I, V, T)=D_{0}[1+a \cdot I(x, t)$ $\left./ I^{*}+b \cdot V(x, t) / V^{*}\right], \rho=I, V$. The second term on the right-hand side of Equations (1) describes the generation of the simplest complexes of radiation defects (divacancies and analogous complexes of radiation 
defects) [7]. The third term on the right-hand side of Equations (1) describes the recombination of point defects.

$$
\begin{aligned}
& \left.\frac{\partial I(x, t)}{\partial x}\right|_{x=0}=0,\left.\frac{\partial V(x, t)}{\partial x}\right|_{x=0}=0,\left.\frac{\partial I(x, t)}{\partial x}\right|_{x=L}=0,\left.\frac{\partial V(x, t)}{\partial x}\right|_{x=L}=0, \\
& I(x, 0)=N_{I} \exp \left[-\frac{\left(x-x_{0 I}\right)^{2}}{x_{I}^{2}}\right], V(x, 0)=N_{V} \exp \left[-\frac{\left(x-x_{0 V}\right)^{2}}{x_{V}^{2}}\right] .
\end{aligned}
$$

We transform Equations (1) to the following integral form:

$$
\left\{\begin{aligned}
I(x, t)=\frac{\partial}{\partial x}\left[\int_{0}^{t} D_{I}(T) \frac{\partial I(x, \tau)}{\partial x} d \tau\right] & -\int_{0}^{t} K_{I}(T) I^{2}(x, \tau) d \tau \\
& -\int_{0}^{t} K_{r}(T) I(x, \tau) V(x, \tau) d \tau+I(x, 0), \\
V(x, t)=\frac{\partial}{\partial x}\left[\int_{0}^{t} D_{V}(T) \frac{\partial V(x, \tau)}{\partial x} d \tau\right] & -\int_{0}^{t} K_{V}(T) V^{2}(x, \tau) d \tau \\
& -\int_{0}^{t} K_{r}(T) I(x, \tau) V(x, \tau) d \tau+V(x, 0) .
\end{aligned}\right.
$$

Now we will solve Equations (1a) by method of averaging of function corrections [10] with decreasing quantity of iteration steps [11]. Framework the approach we used solutions of linear equations (1) with averaged diffusion coefficients $D_{0 I V}$ as initial-order approximations of solutions of Equations (1a). These initial-order approximations could be solved by standard Fourier approach $[12,13]$ and could be written as

$$
\begin{gathered}
I_{0}(r, z, t)=F_{0 I}+\sum_{n=1}^{\infty} F_{n I} J_{0}\left(\frac{r}{R}\right) \cos \left(\frac{\pi n z}{L}\right) \exp \left(-\frac{\pi^{2} n^{2} D_{0 I} t}{L^{2}}\right) \\
V_{0}(r, z, t)=F_{0 V}+\sum_{n=1}^{\infty} F_{n V} J_{0}\left(\frac{r}{R}\right) \cos \left(\frac{\pi n z}{L}\right) \exp \left(-\frac{\pi^{2} n^{2} D_{0 V} t}{L^{2}}\right) .
\end{gathered}
$$


Here $\quad F_{n I}=\frac{2}{L} \int_{0}^{L} I(u, 0) \cos \left(\frac{\pi n u}{L}\right) d u, F_{n V}=\frac{2}{L} \int_{0}^{L} V(u, 0) \cos \left(\frac{\pi n u}{L}\right) d u$.

Substitution of the above series into Equations (1a) gives us a possibility to obtain the first-order approximations of concentrations of point radiation defects in the following form:

$$
\begin{aligned}
& I_{1}(x, t)=-\frac{\pi}{L} \sum_{n=1}^{\infty} n F_{n I} \sin \left(\frac{\pi n x}{L}\right)\left[\int_{0}^{t} D_{I}(T) \exp \left(-\frac{\pi^{2} n^{2} D_{0 I} \tau}{L^{2}}\right) d \tau\right] \\
& -\int_{0}^{t} K_{I}(T)\left[\sum_{n=1}^{\infty} \cos \left(\frac{\pi n x}{L}\right) F_{n I} \exp \left(-\frac{\pi^{2} n^{2} D_{0 I} \tau}{L^{2}}\right)+\frac{F_{0 I}}{2}\right]^{2} d \tau \\
& -\int_{0}^{t} K_{r}(T)\left[\frac{F_{0 I}}{2}+\sum_{n=1}^{\infty} F_{n I} \cos \left(\frac{\pi n x}{L}\right) \exp \left(-\frac{\pi^{2} n^{2} D_{0 I} \tau}{L^{2}}\right)\right] \\
& \times\left[\frac{F_{0 V}}{2}+\sum_{n=1}^{\infty} F_{n V} \cos \left(\frac{\pi n x}{L}\right) \exp \left(-\frac{\pi^{2} n^{2} D_{0 V} \tau}{L^{2}}\right)\right] d \tau+I(x, 0) \\
& V_{1}(x, t)=-\frac{\pi}{L} \sum_{n=1}^{\infty} n F_{n V} \sin \left(\frac{\pi n x}{L}\right)\left[\int_{0}^{t} D_{V}(T) \exp \left(-\frac{\pi^{2} n^{2} D_{0 V} \tau}{L^{2}}\right) d \tau\right] \\
& -\int_{0}^{t} K_{V}(T)\left[\sum_{n=1}^{\infty} \cos \left(\frac{\pi n x}{L}\right) F_{n V} \exp \left(-\frac{\pi^{2} n^{2} D_{0 V} \tau}{L^{2}}\right)+\frac{F_{0 V}}{2}\right]^{2} d \tau \\
& -\int_{0}^{t} K_{r}(T)\left[\frac{F_{0 I}}{2}+\sum_{n=1}^{\infty} F_{n I} \cos \left(\frac{\pi n x}{L}\right) \exp \left(-\frac{\pi^{2} n^{2} D_{0 I} \tau}{L^{2}}\right)\right] \\
& \times\left[\frac{F_{0 V}}{2}+\sum_{n=1}^{\infty} F_{n V} \cos \left(\frac{\pi n x}{L}\right) \exp \left(-\frac{\pi^{2} n^{2} D_{0 V} \tau}{L^{2}}\right)\right] d \tau+V(x, 0)
\end{aligned}
$$


We determine the second- and highest-orders of approximations of concentrations of point radiation defects framework standard iterative procedure of method of averaging of function corrections [10]. In this case $n$-th order approximations of concentrations of defects will be determined by following replacement $I(x, t) \rightarrow \alpha_{n I}+I_{n-1}(x, t), V(x, t) \rightarrow \alpha_{n V}+V_{n-1}(x, t)$ in right sides of Equations (1a), where $\alpha_{n I}$ and $\alpha_{n V}$ are the average values of the considered approximations. In this situation, the secondorder approximations of concentrations of point defects could be written as

$$
\left\{\begin{aligned}
I_{2}(x, t)=\frac{\partial}{\partial x}\left[\int_{0}^{t} D_{I}(T) \frac{\partial I_{1}(x, \tau)}{\partial x} d \tau\right] & -\int_{0}^{t} K_{r}(T)\left[\alpha_{2 I}+I_{1}(x, \tau)\right]\left[\alpha_{2 V}+V_{1}(x, \tau)\right] d \tau \\
& -\int_{0}^{t} K_{I}(T)\left[\alpha_{2 I}+I_{1}(x, \tau)\right]^{2} d \tau+I(x, 0), \\
V_{2}(x, t)=\frac{\partial}{\partial x}\left[\int_{0}^{t} D_{V}(T) \frac{\partial V_{1}(x, \tau)}{\partial x} d \tau\right] & -\int_{0}^{t} K_{r}(T)\left[\alpha_{2 I}+I_{1}(x, \tau)\right]\left[\alpha_{2 V}+V_{1}(x, \tau)\right] d \tau \\
& -\int_{0}^{t} K_{V}(T)\left[\alpha_{2 V}+V_{1}(x, \tau)\right]^{2} d \tau+V(x, 0) .
\end{aligned}\right.
$$

Average values of the second-order approximations of the considered concentrations could be determined by the following standard relations:

$$
\alpha_{2 I}=\frac{1}{\Theta L} \int_{0}^{\Theta} \int_{0}^{L}\left[I_{2}(x, t)-I_{1}(x, t)\right] d x d t, \alpha_{2 V}=\frac{1}{\Theta L} \int_{0}^{\Theta} \int_{0}^{L}\left[V_{2}(x, t)-V_{1}(x, t)\right] d x d t,
$$

where $\Theta$ is the continuance of observation of the change in the concentration of defects with time. 
Substitution of the appropriate approximations of concentrations of point defects into above relations leads to the following relations:

$$
\begin{aligned}
\alpha_{2 V}= & -\frac{\alpha_{2 I}^{2} A_{I 000}+\alpha_{2 I}\left(2 A_{I 010}+A_{r 101}+1\right)+\left(A_{I 010}+A_{r 111}+A_{I 120}\right)}{\left(\alpha_{2 I} A_{r 100}+A_{r 110}\right)} \\
\alpha_{2 I}= & \sqrt[3]{\sqrt{\frac{1}{27}\left(\frac{B_{2}}{B_{4}}-\frac{B_{3}^{2}}{3 B_{4}^{2}}\right)^{3}}-\frac{B_{3}^{3}}{27 B_{4}^{3}}+\frac{B_{2} B_{3}}{6 B_{4}^{2}}-\frac{B_{0}}{2 B_{4}}} \\
& -\sqrt[3]{\sqrt{\frac{1}{27}\left(\frac{B_{2}}{B_{4}}-\frac{B_{3}^{2}}{3 B_{4}^{2}}\right)^{3}}+\frac{B_{3}^{3}}{27 B_{4}^{3}}-\frac{B_{2} B_{3}}{6 B_{4}^{2}}+\frac{B_{0}}{2 B_{4}}}
\end{aligned}
$$

Here $A_{\rho i j k}=\frac{1}{\Theta L} \int_{0}^{\Theta}(\Theta-t) \int_{0}^{L} K_{\rho}^{i}(T) I_{1}^{j}(x, t) V_{1}^{k}(x, t) d x d t, B_{4}=A_{I 000}\left(A_{I 000}\right.$

$\left.\times A_{V 000}-A_{r 100}^{2}\right), B_{3}=A_{I 000}\left\{2 A_{V 000}\left(2 A_{I 010}+A_{r 101}+1\right)-A_{r 100}^{2}\left(2 A_{I 010}\right.\right.$

$\left.\left.+A_{r 101}+1\right)\left[A_{r 110} A_{r 100}+A_{r 100}\left(2 A_{V 010}+A_{r 101}+1\right)\right]+A_{r 100}^{2} A_{r 110}\right\}$,

$B_{2}=A_{V 000}\left[\left(2 A_{I 010}+A_{r 101}+1\right)^{2}+A_{I 000}\left(A_{I 010}+A_{r 111}+A_{I 120}\right)\right]-\left(2 A_{V 010}\right.$

$\left.+A_{r 101}+1\right) A_{I 000} A_{r 110}-\left(2 A_{I 010}+A_{r 101}+1\right)\left[A_{r 110} A_{r 100}+A_{r 100}\left(2 A_{V 010}\right.\right.$

$\left.\left.+A_{r 101}+1\right)\right]-\left(A_{V 010}+A_{r 111}+A_{V 120}\right) A_{r 100}^{2}-A_{r 100} A_{r 100}\left(A_{I 010}+A_{r 111}\right.$

$\left.+A_{I 120}\right)-2 A_{r 110}^{2} A_{r 100}, B_{1}=A_{V 000}\left(2 A_{I 010}+A_{r 101}+1\right)\left(A_{I 010}+A_{r 111}\right.$

$\left.+A_{I 120}\right)-A_{r 110}\left(2 A_{I 010}+A_{r 101}+1\right)\left(2 A_{V 010}+A_{r 101}+1\right)-2 A_{r 100}\left(A_{V 010}\right.$

$\left.+A_{r 111}+A_{V 120}\right) A_{r 110}-A_{r 110} A_{r 110}^{2}-\left(A_{I 010}+A_{r 111}+A_{I 120}\right)\left[A_{r 110} A_{r 100}\right.$

$\left.+A_{r 100}\left(2 A_{V 010}+A_{r 101}+1\right)\right], B_{0}=A_{V 000}\left(A_{I 010}+A_{r 111}+A_{I 120}\right)^{2}-A_{r 110}$

$\left(A_{I 010}+A_{r 111}+A_{I 120}\right)\left(2 A_{V 010}+A_{r 101}+1\right)-\left(A_{V 010}+A_{r 111}+A_{V 120}\right)$

$A_{r 110}^{2}$.

Equations for concentrations of simplest complexes of point defects (divacancies $\Phi_{V}(x, t)$ and diinterstitials $\Phi_{I}(x, t)$ ) could be written as 


$$
\left\{\begin{array}{l}
\frac{\partial \Phi_{I}(x, t)}{\partial t}=\frac{\partial}{\partial x}\left[D_{\Phi_{I}}(T) \frac{\partial \Phi_{I}(x, t)}{\partial x}\right]+k_{I, I}(T) I^{2}(x, t)-k_{I}(T) I(x, t), \\
\frac{\partial \Phi_{V}(x, t)}{\partial t}=\frac{\partial}{\partial x}\left[D_{\Phi_{V}}(T) \frac{\partial \Phi_{V}(x, t)}{\partial x}\right]+k_{V, V}(T) V^{2}(x, t)-k_{V}(T) V(x, t),
\end{array}\right.
$$

with boundary and initial conditions

$$
\begin{aligned}
& \left.\frac{\partial \Phi_{I}(x, t)}{\partial x}\right|_{x=0}=0,\left.\frac{\partial \Phi_{V}(x, t)}{\partial x}\right|_{x=0}=0,\left.\frac{\partial \Phi_{I}(x, t)}{\partial x}\right|_{x=L}=0,\left.\frac{\partial \Phi_{V}(x, t)}{\partial x}\right|_{x=L}=0 \\
& \Phi_{I}(x, 0)=N_{\Phi_{I}} \exp \left[-\frac{\left(x-x_{0 \Phi_{I}}\right)^{2}}{x_{\Phi_{I}}^{2}}\right], \Phi_{V}(x, 0)=N_{\Phi_{V}} \exp \left[-\frac{\left(x-x_{0 \Phi_{V}}\right)^{2}}{x_{\Phi_{V}}^{2}}\right] .
\end{aligned}
$$

Here $D_{\Phi \rho}(T)$ are the diffusion coefficients of the above complexes of radiation defects; $k_{\rho}(T)$ are the parameters of decay of the above complexes. To simplify solution of the above equations we transform them to the following integral form:

$$
\left\{\begin{aligned}
\Phi_{I}(x, t)=\frac{\partial}{\partial x} \int_{0}^{t} D_{\Phi_{I}}(T) \frac{\partial \Phi_{I}(x, \tau)}{\partial x} d \tau & -\int_{0}^{t} k_{I}(T) I(x, \tau) d \tau+\Phi_{I}(x, 0) \\
& +\int_{0}^{t} k_{I, I}(T) I^{2}(x, \tau) d \tau, \\
\Phi_{V}(x, t)=\frac{\partial}{\partial x} \int_{0}^{t} D_{\Phi_{V}}(T) \frac{\partial \Phi_{V}(x, \tau)}{\partial x} d \tau & -\int_{0}^{t} k_{V}(T) V(x, \tau) d \tau+\Phi_{V}(x, 0) \\
& +\int_{0}^{t} k_{V, V}(T) V^{2}(x, \tau) d \tau .
\end{aligned}\right.
$$

Now we solve systems of equations (3a) by method of averaging of function corrections with decreasing quantity of iteration steps. Framework the approach we used solutions of linear equations (3) with averaged diffusion coefficients $D_{0 I V}$ as initial-order approximations of 
solutions of equations (3a). These initial-order approximations could be solved by standard Fourier approach and could be written as

$$
\begin{gathered}
\Phi_{I 0}(x, t)=\frac{F_{0 \Phi_{I}}}{2}+\sum_{n=1}^{\infty} F_{n \Phi_{I}} \cos \left(\frac{\pi n x}{L}\right) \exp \left(-\frac{\pi^{2} n^{2} D_{0 \Phi_{I}} t}{L^{2}}\right), \\
\Phi_{V 0}(x, t)=\frac{F_{0 \Phi_{V}}}{2}+\sum_{n=1}^{\infty} F_{n \Phi_{V}} \cos \left(\frac{\pi n x}{L}\right) \exp \left(-\frac{\pi^{2} n^{2} D_{0 \Phi_{V}} t}{L^{2}}\right) .
\end{gathered}
$$

Here $F_{n \Phi_{I}}=\frac{2}{L} \int_{0}^{L} \Phi_{I}(\nu, 0) \cos \left(\frac{\pi n \nu}{L}\right) d \nu, F_{n \Phi_{I}}=\frac{2}{L} \int_{0}^{L} \Phi_{I}(\nu, 0) \cos \left(\frac{\pi n \nu}{L}\right) d \nu$.

Substitution of the above series into Equations (3a) gives us a possibility to obtain the first-order approximations of concentrations of point radiation defects in the following form:

$$
\begin{aligned}
\Phi_{1 I}(x, t)= & -\frac{\pi}{L} \sum_{n=1}^{\infty} F_{n \Phi_{I}} \sin \left(\frac{\pi n x}{L}\right) \int_{0}^{t} D_{\Phi_{I}}(T) \exp \left(-\frac{\pi^{2} n^{2} D_{0 \Phi_{I}} \tau}{L^{2}}\right) d \tau \\
& -\int_{0}^{t} k_{I}(T)\left[\frac{F_{0 \Phi_{I}}}{2}+\sum_{n=1}^{\infty} F_{n \Phi_{I}} \cos \left(\frac{\pi n x}{L}\right) \exp \left(-\frac{\pi^{2} n^{2} D_{0 \Phi_{I}} \tau}{L^{2}}\right)\right] d \tau \\
+ & \int_{0}^{t} k_{I, I}(T)\left[\sum_{n=1}^{\infty} F_{n \Phi_{I}} \cos \left(\frac{\pi n x}{L}\right) \exp \left(-\frac{\pi^{2} n^{2} D_{0 \Phi_{I}} \tau}{L^{2}}\right)+\frac{1}{2} F_{0 \Phi_{I}}\right]^{2} d \tau, \\
\Phi_{1 V}(x, t)= & -\frac{\pi}{L} \sum_{n=1}^{\infty} F_{n \Phi_{V}} \sin \left(\frac{\pi n x}{L}\right) \int_{0}^{t} D_{\Phi_{V}}(T) \exp \left(-\frac{\pi^{2} n^{2} D_{0 \Phi_{V}} \tau}{L^{2}}\right) d \tau \\
& -\int_{0}^{t} k_{V}(T)\left[\frac{F_{0 \Phi_{V}}}{2}+\sum_{n=1}^{\infty} F_{n \Phi_{V}} \cos \left(\frac{\pi n x}{L}\right) \exp \left(-\frac{\pi^{2} n^{2} D_{0 \Phi_{V}} \tau}{L^{2}}\right)\right] d \tau \\
+ & \int_{0}^{t} k_{V, V}(T)\left[\sum_{n=1}^{\infty} F_{n \Phi_{V}} \cos \left(\frac{\pi n x}{L}\right) \exp \left(-\frac{\pi^{2} n^{2} D_{0 \Phi_{V}} \tau}{L^{2}}\right)+\frac{1}{2} F_{0 \Phi_{V}}\right]^{2} d \tau .
\end{aligned}
$$


We determine the second- and highest-orders of approximations of concentrations of simplest complexes of point radiation defects framework standard iterative procedure of method of averaging of function corrections [10]. In this case $n$-th order approximations of concentrations of complexes of defects will be determined by following replacement $\Phi_{I}(x, t) \rightarrow \alpha_{n \Phi I}+\Phi_{I n-1}(x, t), \Phi_{V}(x, t) \rightarrow \alpha_{n \Phi V}+\Phi_{V n-1}(x, t)$ in right sides of Equations (3a), where $\alpha_{n I}$ and $\alpha_{n V}$ are the average values of the considered approximations. In this situation, the second-order approximations of concentrations of complexes of point defects could be written as

$$
\left\{\begin{aligned}
\Phi_{2 I}(x, t)=\frac{\partial}{\partial x} \int_{0}^{t} D_{\Phi_{I}}(T) \frac{\partial \Phi_{1 I}(x, \tau)}{\partial x} d \tau & +\int_{0}^{t} k_{I, I}(T) I^{2}(x, \tau) d \tau \\
& -\int_{0}^{t} k_{I}(T) I(x, \tau) d \tau+\Phi_{I}(x, 0), \\
\Phi_{2 V}(x, t)=\frac{\partial}{\partial x} \int_{0}^{t} D_{\Phi_{V}}(T) \frac{\partial \Phi_{1 V}(x, \tau)}{\partial x} d \tau & +\int_{0}^{t} k_{V, V}(T) V^{2}(x, \tau) d \tau \\
& -\int_{0}^{t} k_{V}(T) V(x, \tau) d \tau+\Phi_{V}(x, 0) .
\end{aligned}\right.
$$

We determine spatio-temporal distribution of temperature, generated during generation of radiation defects due to radiation processing, by solution of the following equation:

$$
c(T) \frac{\partial T(x, t)}{\partial t}=\frac{\partial}{\partial x}\left[\lambda(T) \frac{\partial T(x, t)}{\partial x}\right] .
$$

Temperature dependence of heat capacitance $c(T)$ could be approximated by the following function: $c(T)=c_{0}\left[1-\exp \left(-T / T_{d}\right)\right]$, where $T_{d}$ is the Debye temperature. If current temperature is larger, than the Debye temperature, heat conduction coefficient could be approximated by the following function: $\lambda(T)=\lambda_{0}\left\{1+\mu\left[T_{d} / T(x, t)\right]^{\varphi}\right\}$. 
In the same area of temperatures one can consider the following relation: $c(T) \approx c_{0}$. The Equation (5) is complemented by the following boundary and initial conditions:

$$
\left.\frac{\partial T(x, t)}{\partial x}\right|_{x=0}=0,\left.\frac{\partial T(x, t)}{\partial x}\right|_{x=L}=0, T(x, 0)=T_{r}
$$

Here $T_{r}$ is the room temperature. With account dependence of heat diffusion coefficient on temperature one can transform the Equation (5) to the following differential (7a) or integral (7b) form

$$
\begin{gathered}
\frac{c_{0}}{\varphi} \frac{\partial T^{\varphi}(x, t)}{\partial t}=\mu \lambda_{0} T_{d}^{\varphi} T(x, t) \frac{\partial^{2} T(x, t)}{\partial x^{2}}-\mu \varphi \lambda_{0} T_{d}^{\varphi}\left[\frac{\partial T(x, t)}{\partial x}\right]^{2} \\
\frac{c_{0}}{\varphi} T^{\varphi}(x, t)=\mu \lambda_{0} T_{d}^{\varphi} \int_{0}^{t} T(x, t) \frac{\partial^{2} T(x, \tau)}{\partial x^{2}} d \tau-\mu \varphi \lambda_{0} T_{d}^{\varphi} \int_{0}^{t}\left[\frac{\partial T(x, \tau)}{\partial x}\right]^{2} d \tau
\end{gathered}
$$

We solved the Equation (7b) by method of averaging of function corrections with decreased quantity of iterative steps. Framework the approach we used solutions of linear equation (5) with averaged heat diffusion coefficients $\lambda_{0}$ as initial-order approximations of solutions of equation (7b). These initial-order approximations could be solved by standard Fourier approach and could be written as

$$
T_{0}(x, t)=\frac{F_{0}}{2}+\sum_{n=1}^{\infty} F_{n} \cos \left(\frac{\pi n x}{L}\right) \exp \left(-\frac{\pi^{2} n^{2} \lambda_{0} t}{L^{2} c_{0}}\right) .
$$

Here $F_{n}=\frac{2}{L} \int_{0}^{L} T(\nu, 0) \cos \left(\frac{\pi n x}{L}\right) d \nu$. Substitution of the above series into equation (7b) gives us a possibility to obtain the first-order approximation of temperature in the following form 


$$
\begin{aligned}
& \frac{c_{0}}{\varphi} T_{1}^{\varphi}(x, t)=-\mu \frac{\pi^{2}}{L^{2}} \int_{0}^{t}\left[\frac{F_{0}}{2}+\sum_{n=1}^{\infty} F_{n} \cos \left(\frac{\pi n x}{L}\right) \exp \left(-\frac{\pi^{2} n^{2} \lambda_{0} \tau}{L^{2} c_{0}}\right)\right] \\
& \times \sum_{n=1}^{\infty} \exp \left(-\frac{\pi^{2} n^{2} \lambda_{0} \tau}{L^{2} c_{0}}\right) d \tau \times n^{2} F_{n} \cos \left(\frac{\pi n x}{L}\right) T_{d}^{\varphi} \\
&-\mu \varphi \lambda_{0} T_{d}^{\varphi} \frac{\pi^{2}}{L^{2}} \int_{0}^{t}\left[\sum_{n=1}^{\infty} n^{2} F_{n} \sin \left(\frac{\pi n x}{L}\right) \exp \left(-\frac{\pi^{2} n^{2} \lambda_{0} \tau}{L^{2} c_{0}}\right) \frac{\partial T(x, \tau)}{\partial x}\right]^{2} d \tau .
\end{aligned}
$$

We determine the second- and highest-orders of approximation of temperature framework standard iterative procedure of method of averaging of function corrections [10]. In this case $n$-th order approximation of temperature will be determined by following replacement $T(x, t) \rightarrow \alpha_{n T}+T_{n-1}(x, t)$ in right sides of Equation (7b), where $\alpha_{n T}$ is the average value of the considered approximation. In this situation, the second-order approximation of temperature could be written as

$$
\begin{aligned}
T_{2}(x, t)=\left\{\mu \lambda_{0} T_{d}^{\varphi} \frac{\varphi}{c_{0}} \int_{0}^{t}\left[\alpha_{2 T}+T_{1}(x, t)\right] \frac{\partial^{2} T_{1}(x, \tau)}{\partial x^{2}} d \tau\right. & \\
& \left.-\mu \lambda_{0} T_{d}^{\varphi} \frac{\varphi^{2}}{c_{0}} \int_{0}^{t}\left[\frac{\partial T_{1}(x, \tau)}{\partial x}\right]^{2} d \tau\right\}^{\frac{1}{\varphi}} .
\end{aligned}
$$

Not yet known average value $\alpha_{2 T}$ by solution the following equation:

$$
\begin{aligned}
\alpha_{2 T}=\int_{0}^{\Theta} \int_{0}^{L}\left[\left\{\mu \lambda_{0} T_{d}^{\varphi}\right.\right. & \frac{\varphi}{c_{0}} \int_{0}^{t}\left[\alpha_{2 T}+T_{1}(x, t)\right] \frac{\partial^{2} T_{1}(x, \tau)}{\partial x^{2}} d \tau \\
& \left.\left.-\mu \lambda_{0} T_{d}^{\varphi} \frac{\varphi^{2}}{c_{0}} \int_{0}^{t}\left[\frac{\partial T_{1}(x, \tau)}{\partial x}\right]^{2} d \tau\right\}^{\frac{1}{\varphi}}-T_{1}(x, t)\right] d x d t .
\end{aligned}
$$

The value of this average value depends on the parameter $\varphi$, determined by the experimental data. 


\section{Discussion}

In this paper, we analyzed spatio-temporal distribution of the concentrations of radiation defects in doped with ion implantation material. Figure 1 shows the distribution of the concentration of point radiation defects for two doses: $5 \cdot 10^{14} \mathrm{~cm}^{-2}$ (curves 1) and $5 \cdot 10^{15} \mathrm{~cm}^{-2}$ (curves 2). The solid lines are the calculated curves, the dashed lines are the experimental curves from [8]. According to the experimental data, the concentration of defects increases to the irradiated sample boundary, while having a maximum in the depth of the sample. Apparently, an increase in the concentration of defects with approach to the irradiated surface is a consequence of a large number of defects in the near-surface region at the initial stage of doping. Over time, the defects recombine among themselves and diffuse from the near-surface region. To account for these changes, the concentration dependence of the defect diffusion coefficient was used.

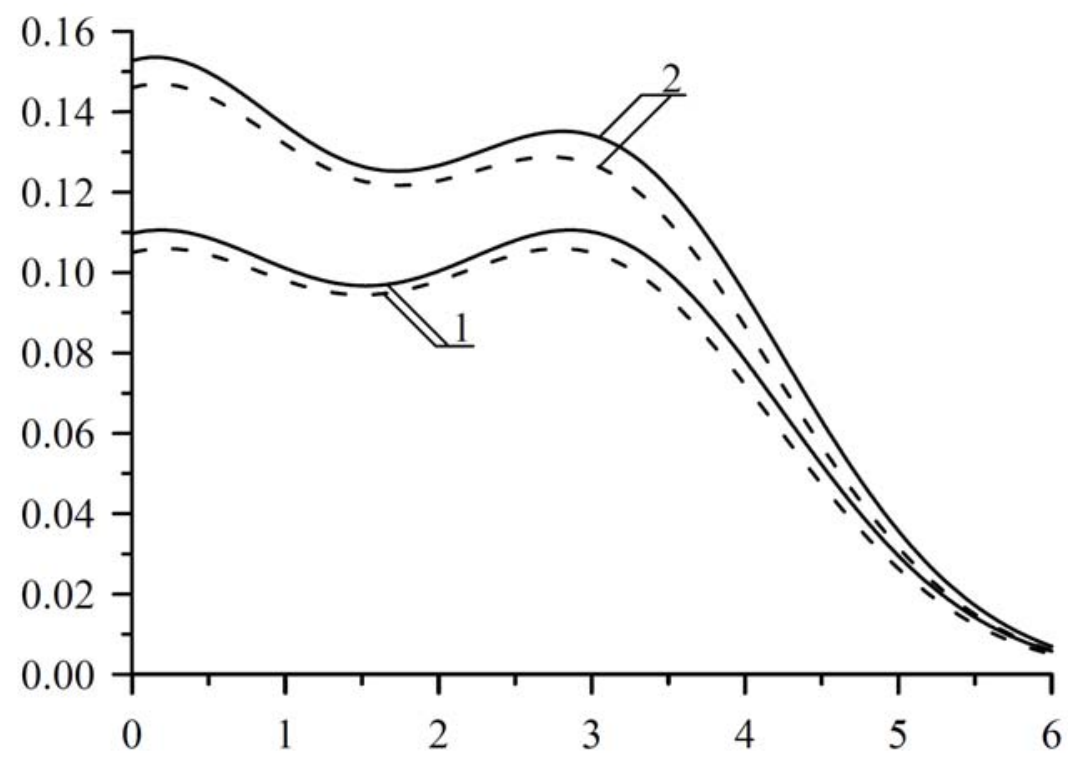

Figure 1. The distribution of the concentration of radiation defects at the dose $5 \cdot 10^{14} \mathrm{~cm}^{-2}$ (curve 1) and the dose $5 \cdot 10^{15} \mathrm{~cm}^{-2}$ (curve 2). Solid curves are the calculated results. Dotted curves are experimental results. 


\section{Conclusion}

In this paper, we introduce a model for the redistribution and interaction of point radiation defects between themselves, as well as their simplest complexes in a material, taking into account the experimentally non-monotonicity of the distribution of the concentration of radiation defects. To take into account this non-monotonicity, the previously used model in the literature for the analysis of spatio-temporal distributions of the concentration of radiation defects was supplemented by the concentration dependence of their diffusion coefficient.

\section{References}

[1] G. A. Tarnavsky and E. V. Vorozhtsov, Dopant implantation into the silicon substrate with non-planar surface, Energy and Power Engineering 2(2) (2010), 73-77.

DOI: https://doi.org/10.4236/epe.2010.22011

[2] V. A. Ivchenko, Development of amorphized states in subsurface metal regions under radiation exposure, Technical Physics Letters 39(4) (2013), 357-359.

DOI: https://doi.org/10.1134/S106378501304007X

[3] V. M. Barbashov and O. A. Kalashnikov, Methods of functional-logic simulation of radiation-induced failures of electronic systems based on the fuzzy state machine model, Russian Microelectronics 46(3) (2017), 155-161.

DOI: https://doi.org/10.1134/S1063739717030027

[4] A. A. Lebedev, A. M. Ivanov and N. B. Strokan, Radiation resistance of SiC and nuclear-radiation detectors based on SiC films, Semiconductors 38(2) (2004), 124-147.

[5] E. V. Kalinina, V. G. Kossov, R. R. Yafaev, A. M. Strel'chuk and G. N. Violina, A high-temperature radiation-resistant rectifier based on $p^{+}-n$ junctions in ${ }_{4} \mathrm{H}-\mathrm{SiC}$ ion-implanted with aluminum, Semiconductors 44(6) (2010), 778-788.

DOI: https://doi.org/10.1134/S1063782610060151

[6] A. A. Lebedev, S. V. Belov, M. G. Mynbaeva, A. M. Strel'chuk, E. V. Bogdanova, Yu. N. Makarov, A. S. Usikov, S. Yu. Kurin, I. S. Barash, A. D. Roenkov and V. V. Kozlovski, Radiation hardness of $n$-GaN schottky diodes, Semiconductors 49(10) (2015), 1341-1343.

DOI: https://doi.org/10.1134/S1063782615100127 
[7] V. L. Vinetskiy and G. A. Kholodar, Radiative Physics of Semiconductors, Naukova Dumka, Kiev, 1979.

[8] N. A. Sobolev, B. Ya. Ber, D. Yu. Kazantsev, A. E. Kalyadin, K. V. Karabeshkin, V. M. Mikushkin, V. I. Sakharov, I. T. Serenkov, E. I. Shek, E. V. Sherstnev and N. M. Shmidt, The effect of dose of nitrogen-ion implantation on the concentration of point defects introduced into GaAs layers, Technical Physics Letters 44(7) (2018), 574-576.

DOI: https://doi.org/10.1134/S1063785018070131

[9] P. M. Fahey, P. B. Griffin and J. D. Plummer, Point defects and dopant diffusion in silicon, Reviews of Modern Physics 61(2) (1989), 289-388.

DOI: https://doi.org/10.1103/RevModPhys.61.289

[10] Yu. D. Sokolov, About the definition of dynamic forces in the mine lifting, Applied Mechanics 1(1) (1955), 23-35.

[11] E. L. Pankratov, Dynamics of $\delta$-dopant redistribution during heterostructure growth, The European Physical Journal B 57(3) (2007), 251-256.

DOI: https://doi.org/10.1140/epjb/e2007-00173-8 